\title{
Bronchopleural fistula in squamous cell lung cancer following anlotinib treatment: A case report
}

\author{
DAN LI ${ }^{1,2^{*}}$, GUOLI WEI ${ }^{1,3^{*}}$, LINGCHANG LI $^{1,3}$, JUN MA $^{1,3}$, XIAOFEI HUANG $^{1,3}$, \\ FENGXIA QIN $^{1,3}$, ZHEN GONG ${ }^{1,3}$ and JIEGE HUO ${ }^{1,3}$ \\ ${ }^{1}$ Department of Oncology, Affiliated Hospital of Integrated Traditional Chinese and Western Medicine, \\ Nanjing University of Chinese Medicine, Nanjing, Jiangsu 210028; ${ }^{2}$ Nanjing University of Chinese Medicine; \\ ${ }^{3}$ Department of Oncology, Jiangsu Province Academy of Traditional Chinese Medicine, Nanjing, Jiangsu 210046, P.R. China
}

Received February 15, 2019; Accepted September 24, 2019

DOI: $10.3892 / \mathrm{mco} .2019 .1939$

\begin{abstract}
Anlotinib is a multi-target tyrosine kinase inhibitor and has been approved for the treatment of patients with advanced non-small cell lung cancer. The most common adverse events of this treatment include hypertension, fatigue, thyroid-stimulating hormone elevation, hypertriglyceridemia, hand-foot syndrome and hypercholesterolemia. The present study reported the case of a 69-year-old man with squamous cell lung cancer that experienced disease progression following first-line and second-line chemotherapy. Subsequently, anlotinib was administered as a third-line therapy. Following the second cycle of oral targeted therapy, the patient was admitted to the hospital with a one-week history of chest tightnesss, shortness of breath and cough blood-stained sputum and necrosis. Computed tomography scan showed: Bronchopleural fistula (BPF) complicating lung cancer. However, symptoms were not relieved following anti-infective treatment and the patient subsequently died of respiratory failure. To the best of our knowledge, this is the first case of bronchopleural fistula associated with the use of anlotinib in a patient with squamous cell lung cancer.
\end{abstract}

\section{Introduction}

Lung cancer is the leading incident cancer and cause of cancer mortality worldwide. There were about 2.1 million new lung cancer cases and 1.8 million deaths predicted in 2018 (1). Unfortunately, there is currently no standard third-line treatment

Correspondence to: Professor Jiege Huo, Department of Oncology, Affiliated Hospital of Integrated Traditional Chinese and Western Medicine, Nanjing University of Chinese Medicine, 100 Cross Street, Maigaoqiao, Nanjing, Jiangsu 210028, P.R. China

E-mail: huojiege@jsatcm.com

${ }^{*}$ Contributed equally

Key words: anlotinib, bronchopleural fistula, tyrosine kinase inhibitor, squamous cell lung cancer for advanced non-small-cell lung cancer (NSCLC). So there is still a need to develop innovative, more effective, and safer anticancer drugs.

Anlotinib is a novel multi-target tyrosine kinase inhibitor that is designed to primarily inhibit vascular endothelial growth factor receptor (VEGFR)2/3, fibroblast growth factor receptor (FGFR)1-4, platelet-derived growth factor receptors (PDGFR) $\alpha / \beta$, c-Kit, and Ret (2). ALTER 0303 Phase 3 Randomized Clinical Trial showed that anlotinib as third-line therapy for advanced NSCLC can prolong progress free survival (PFS) for 4 months $(5.4$ vs. 1.4, $\mathrm{P}<0.0001)$ and $\mathrm{OS}$ for 3.3 months (9.6 vs. $6.3, \mathrm{P}=0.002$ ) compared with placebo among Chinese patients. A total of 437 patients were randomized 2:1 to receive either oral anlotinib or placebo (12 mg QD from days 1 to 14 of a 21-day cycle). Treatment continued until tumor progression or discontinuation due to toxicity. An exploratory subgroup analysis of the ALTER0303 trial showed that anlotinib significantly improved progress free survival (PFS) and overall survival (OS) in patients with both sensitive EGFR mutations and wild-type EGFR and it can prolong PFS for 2.93 months ( $\mathrm{P}=0.001)$ and $\mathrm{OS}$ for 2 months $(\mathrm{P}=0.0932)$ for squamous lung cancer. It is the first oral multi-target tyrosine kinase inhibitor drug can prolong PFS for squamous lung cancer (3). Moreover, The China Food and Drug Administration (CFDA) approved single agent anlotinib as a third-line treatment for patients with advanced NSCLC. Clinical trials have confirmed that anlotinib is well tolerated. Wu et al (4) assessed the efficacy and toxicity of anlotinib. They found that anlotinib plays a significant role in the salvage treatment of advanced NSCLC.

Anlotinib can inhibit both tumor angiogenesis and tumor cell proliferation (5). Compared with other RTK inhibitors, such as sorafenib and sunitinib, anlotinib can inhibit more targets. Studies have shown that anlotinib significantly inhibits VEGF/PDGF-BB/FGF-2-induced angiogenesis in vitro and in vivo (2). Thence, it had broader and better antitumor efficacy than did other RTK inhibitors in vivo. There are also many studies on the anti-tumor mechanism of anlotinib. One study showed that anlotinib could induce protective autophagy, related to anti-angiogenic property of multikinase inhibitor anlotinib (6). Lu et al (7) believe that anlotinib-induced serum CCL2 level decreases were associated with the benefits of PFS and OS in refractory advanced NSCLC patients. 

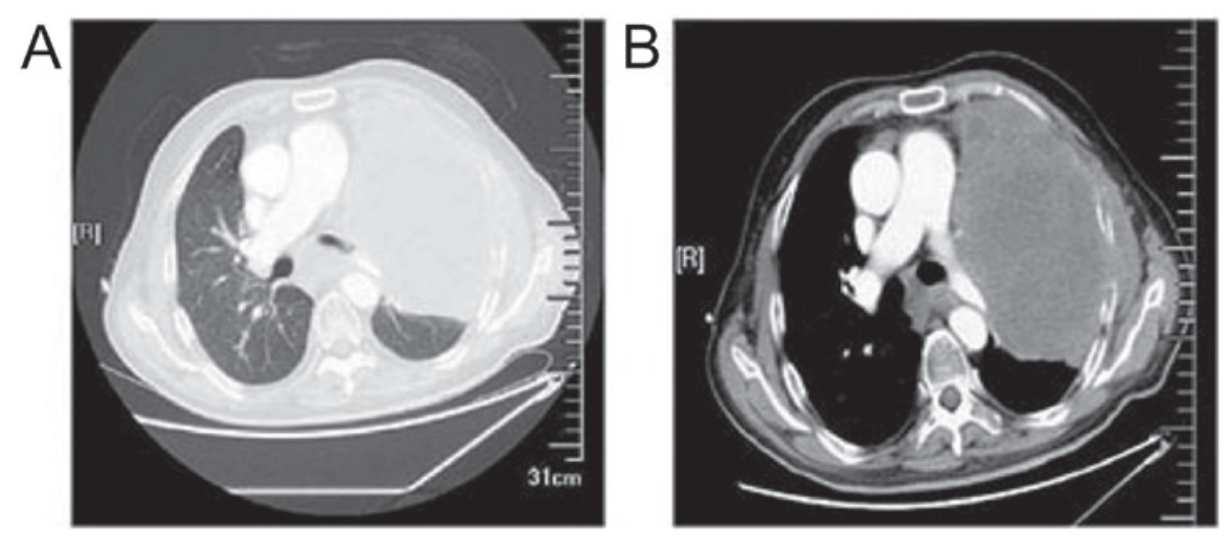

Figure 1. Chest CT scan demonstrating lung cancer with multiple lung, lymph node metastasis and ribs destruction. (A) Window of lung fields. (B) Mediastinal windows. CT, computed tomography.
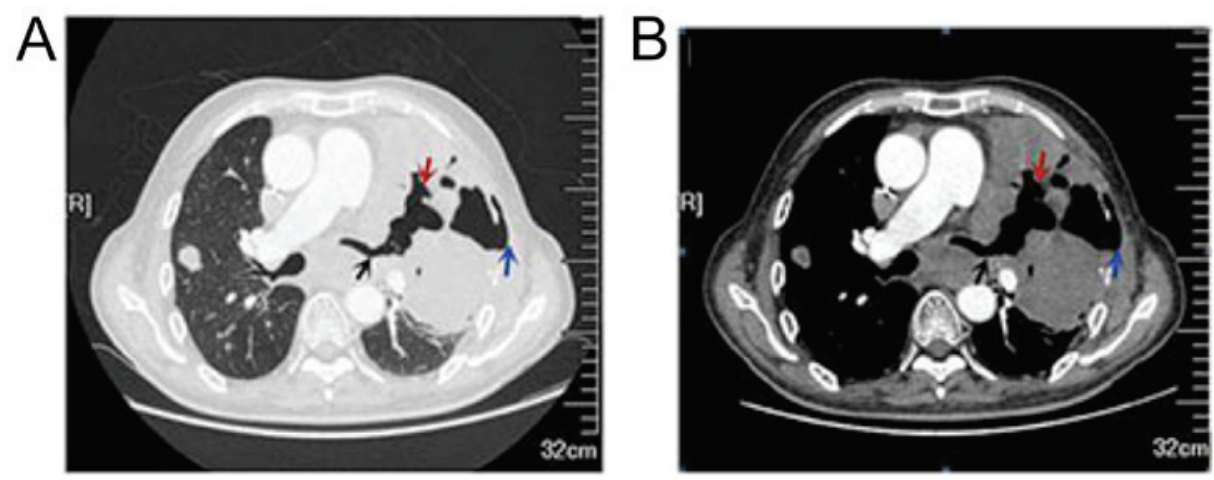

Figure 2. Chest CT scan demonstrating chest wall emphysema (blue arrows), bronchus fistula fistula (black arrows) and lung lesions necrotic cavity (red arrows). (A) Window of lung fields. (B) Mediastinal windows. CT, computed tomography.

Compared to the mono-target agents, it may cause more AEs in patients. Whether side effects occur dependent on the individual physical characteristics of the patients (8). The most common adverse events (AEs) of anlotinib included hypertension, fatigue, lack or loss of appetite, thyroid-stimulating hormone elevation, anorexia hypertriglyceridemia, hand-foot syndrome and hypercholesterolemia (3). However, bronchopleural fistula as a result of treatment with anlotinib or other TKIs have not been reported. Here, we present the first report of a bronchopleural fistula caused by multi-target tyrosine kinase inhibitor therapy.

\section{Case report}

A 69-year-old male never-smoker was admitted to the hospital in April 2011 with a cough and hemoptysis, which had been ongoing for 3 months. The vital signs of the patient were stable. Enhanced computed tomography (CT) scans of the patient's chest revealed tumors on the lower lobe of the right lung and on the upper lobe of the left lung. Then he underwent radical resection of the right lung cancer and nodule wedge resection of left lung. Postoperative pathology: Poorly differentiated squamous cell carcinoma in the right lung and left lung. The patient was diagnosed as having stage IA cancer (cT1cNOM0) according to postoperative pathology, and began receiving chemotherapy consisting of gemcitabine and cisplatin for four cycles. However, a chest CT scan demonstrated disease progression with metastases in the lungs in December 2016. Therefore, he received first-line chemotherapy including paclitaxel liposome $(210 \mathrm{mg})$ and nedaplatin $(100 \mathrm{mg})$ for 6 cycles from July 1, 2017 to October 24, 2017. In January 3th, 2018, efficacy evaluation is disease progression (PD,RECIST1.1). To control the disease second-line chemotherapy GP regimen (gemcitabine $1.4 \mathrm{~g} \mathrm{~d} 1$, d8+ nedaplatin $100 \mathrm{mg} \mathrm{d} 1)$ treatment was given for 2 months. He received 'gemcitabine $1.4 \mathrm{~g} \mathrm{~d} 1$, d8+ nedaplatin $100 \mathrm{mg}$ d1' second-line chemotherapy for 2 cycles from January 2018 to March 2018. Efficacy evaluation is stable disease (SD, RECIST1.1) in this time, but patients refused to continue to receive chemotherapy due to gastrointestinal reaction (NCI CTC3.0 grade III). In June 2018, the CT of the chest was obtained. This revealed the two lungs metastasized, the left lung lesions increased, the two lung metastases were larger than the front, and the mediastinal multiple lymph node metastasis lesions were both larger than the previous one. The left 4 and 5 ribs were invaded by tumor, no abnormality in the abdominal pelvic CT (Fig. 1A-B). Efficacy evaluation is disease progression (PD, RECIST1.1). We recommended he to biopsy again for genetic testing to see if there were sensitive mutations, but he refused. Considered the poor general condition of this patient (PS score 2), anlotinib was planned as third-line therapy (12 mg, po, qd, d1-14, q21 days). Initially the patient was well tolerated. After the second cycle of oral targeted therapy, the patient presented to hospital with pain in the oral mucosa and tongue, which 
affected eating. He also felt pleuritic chest pain, shortness of breath, and nonproductive cough which was significantly worse than before. Chest CT demonstrated left lung lesions appeared necrotic cavity with left chest wall emphysema and the left upper lobe and the lower lobe of the two lungs showed a exudative change (Fig. 2A-B), the right upper lobe subpleural lesions was smaller than before. Efficacy evaluation is stable disease (SD, RECIST1.1). The cardiothoracic surgery was consulted, BPF caused by targeted treatment was considered comprehensively, and then brought about secondary infection. Anlotinib was discontinued. However, the symptoms cannot be relieved after anti-infective treatment and supportive therapies. Finally, the patient died of respiratory failure on August 28,2018 . The overall survival time was 88 months and it was found to survive for 22 months after lung metastasis.

\section{Discussion}

Anlotinib is a newly developed multiple receptor tyrosine kinase (RTKs) oral small molecule inhibitor, and is recommended in the Chinese Society of Clinical Oncology Guidelines for the Diagnosis and Treatment of Primary Lung Cancer (2018 Edition) for the same indication (9). It inhibits both neoplastic angiogenesis and tumor growth pathways and is well tolerated.

Bronchopleural fistula (BPF) is defined as an abnormal channel that develops as a result of connection between the bronchus and the pleura. It may be caused by necrotizing pneumonia or empyema, lung tumor, lung injury, chest drainage and chest puncture, radiotherapy and chemotherapy. It is most commonly occur after pneumonectomy. Residual tumor in the bronchial stump after resection, inflammation of the residual site, or combined with tuberculosis, follow by chemotherapy and radiotherapy will increase the incidence of bronchopleural fistula. In addition, there is a meta analysis show that diabetes mellitus is an independent risk factor of bronchopleural fistula after pulmonary resection (10). Bronchopleural fistula (BPF) is a rare but severe complication, it has a higher mortality rate. It is reported that the mortality rate of BPF after pneumonectomy is $18-50 \%$ (11). Chang et al reported 1 patient (1.6\%) complicated with bronchial fistula in 64 cases of patients with unresectable III stage non-small cell lung cancer after proton beam radiotherapy (12). Zheng et al (13) reported 2 of 682 patients $(0.29 \%)$ with lung cancer after pulmonary ablation complicated with BPF (13). At present, there is no relevant literature report about the occurrence of BPF after targeted therapy in patients with primary lung cancer. In the ALTER 0303 study, no patient experienced adverse events of bronchopleural fistula. Only a small proportion of subjects reported grades $3 / 4$ event (3). Thus, BPF may be rare in the general population. No cases have been reported.

The mechanism by which targeted drugs cause perforation of lung cancer is not clear. There are more reports of targeted drugs causing gastrointestinal perforation (14-17). However, reports of targeted drug-induced perforation of the lungs are rare, especially in primary lung cancer. Gennatas et al (18) reported a case of pneumothorax during crizotinib therapy in a patient with with a Stage IV, TTF1 positive, EGFR wild-type adenocarcinoma of the lung. Pneumothorax occurring in the setting of targeted therapy for pulmonary metastatic disease is a well-described clinical situation that has been reviewed by previous authors (19-22). Chi et al (23) reported 166 patients with Refractory Metastatic Soft-Tissue Sarcoma were treated with anlotinib. What needs to be noticed is that mong those, there were 4 patients (2.4\%) with grades 3 pneumothorax. But no treatment-related death occurred. We think there are two reasons of BPF for this patient. Firstly, the tumor was very large, connected with the main bronchus and invaded the pleura, which provided anatomical and pathological basis for the formation of BPF. Direct invasion of tumor or extension of cavitary tumor lesions could be the probable causes. Secondly, anlotinib can inhibit tumor angiogenesis and tumor growth. We also found clinically in other patients that the probability of tumor necrosis after treatment with erlotinib was significantly higher than other TKI-targeted drugs. The tumor of this patient shrank, and the necrosis inside the tumor was obviously and accompanied by cavitation after oral administration of anlotinib, which eventually led to the occurrence of bronchopleural fistula.

To the best of our knowledge, this is the first known case report of a bronchopleural fistula caused by multi-target tyrosine kinase inhibitor therapy. Despite there is no bronchopleural fistula complication found in the patient in ALTER 0303 study, we should be careful for patients with non-small-cell lung cancer, especially centrally located squamous cell carcinoma, large tumor, close to trachea and/or pleura, and combined with diabetes mellitus, when we use the drugs with antiangiogenic effect, especially for squamous cell lung cancer. Because they have the potential to cause life-threatening complications, like bronchopleural fistula.

\section{Acknowledgements}

Not applicable.

\section{Funding}

The present study was supported by the Project of Nanjing Science and Technology Commission of Jiangsu Province (grant no. 201605064) and Jiangsu Provincial Department of Education (grant no.SJCX-0374).

\section{Availability of data and materials}

The datasets used and/or analyzed during the present study are available from the corresponding author on reasonable request.

\section{Authors' contributions}

JGH conceived and designed the study. DL and GLW wrote the manuscript. GLW, LCL, JM, XFH, FXQ and ZG have contributed to the clinical management of the patient. All authors critically reviewed the manuscript and approved the final manuscript.

\section{Ethics approval and consent to participate}

The China Food and Drug Administration (CFDA) approved single agent anlotinib as a third-line treatment for patients with advanced NSCLC. The current study was approved by 
the Institutional Ethics Review Board of Jiangsu Province Academy of Traditional Chinese Medicine, China Nanjing 220000. The patient and his family agreed to participate in this study. This study is normative.

\section{Patient consent for publication}

The patient and his family agreed to the publication of the case details.

\section{Competing interests}

The authors declare that they have no competing interests.

\section{References}

1. Bray F, Ferlay J, Soerjomataram I, Siegel RL, Torre LA and Jemal A: Global cancer statistics 2018: GLOBOCAN estimates of incidence and mortality worldwide for 36 cancers in 185 countries. CA Cancer J Clin 68: 394-424, 2018.

2. Lin B, Song X, Yang D, Bai D, Yao Y and Lu N: Anlotinib inhibits angiogenesis via suppressing the activation of VEGFR2, PDGFR $\beta$ and FGFR1. Gene 654: 77-86, 2018.

3. Han BH, Li K, Wang Q, Zhang L, Shi J, Wang Z, Cheng Y, He J, Shi Y, Zhao Y, et al: Effect of Anlotinib as a third-line or further treatment on overall survival of patients with advanced non-small cell lung cancer: The ALTER 0303 phase 3 randomized clinical trial. JAMA Oncol 4: 1569-1575, 2018.

4. Wu D, Nie J, Dai L, Hu W, Zhang J, Chen X, Ma X, Tian G, Han J, Han S, et al: Salvage treatment with anlotinib for advanced non-small cell lung cancer. Thorac Cancer 10: 1590-1596, 2019.

5. Xie C, Wan X, Quan H, Zheng M, Fu L, Li Y and Lou L: Preclinical characterization of anlotinib, a highly potent and selective vascular endothelial growth factor receptor-2 inhibitor. Cancer Sci 109: 1207-1219, 2018.

6. Liang L, Hui K, Hu C, Wen Y, Yang S, Zhu P, Wang L, Xia Y, Qiao Y, Sun W, et al: Autophagy inhibition potentiates the anti-angiogenic property of multikinase inhibitor anlotinib through JAK2/STAT3/VEGFA signaling in non-small cell lung cancer cells. J Exp Clin Cancer Res 38: 71, 2019.

7. Lu J, Zhong H, Chu T, Zhang X, Li R, Sun J, Zhong R, Yang Y, Alam MS, Lou Y, et al: Role of anlotinib-induced CCL2 decrease in anti-angiogenesis and response prediction for nonsmall cell lung cancer therapy. Eur Respir J 53, 2019.

8. Wang J, Zhao Y, Wang Q, Zhang L, Shi J, Wang Z, Cheng Y, He J, Shi Y, Yu H, et al: Prognostic factors of refractory NSCLC patients receiving anlotinib hydrochloride as the third- or further-line treatment. Cancer Biol Med 15: 443-451, 2018.

9. No authors listed: Chinese guidelines for diagnosis and treatment of primary lung cancer 2018 (English version). Chin J Cancer Res 31: 1-28, 2019.
10. Li SJ, Fan J, Zhou J, Ren YT, Shen C and Che GW: Diabetes mellitus and risk of bronchopleural fistula after pulmonary resections: A meta-analysis. Ann Thorac Surg 102: 328-339, 2016.

11. Okuda M, Go T and Yokomise H: Risk factor of bronchopleural fistula after general thoracic surgery: Review article. Gen Thorac Cardiovasc Surg 65: 679-685, 2017.

12. Chang JY, Verma V, Li M, Zhang W, Komaki R, Lu C, Allen PK, Liao Z, Welsh J, Lin SH, et al: Proton beam radiotherapy and concurrent chemotherapy for unresectable stage III non-small cell lung cancer: Final results of a phase 2 study. JAMA Oncol 3: e172032, 2017.

13. Zheng A, Yang X, Ye X, Huang G, Wei Z, Wang J, Han X, Ni X and Meng M: Bronchopleural fistula after lung ablation: Experience in two cases and literature review. Indian J Cancer 52 (Suppl 2): e41-e46, 2015.

14. Peters NA, Richel DJ, Verhoeff JJ and Stalpers LJ: Bowel perforation after radiotherapy in a patient receiving sorafenib. J Clin Oncol 26: 2405-2406, 2008.

15. Inoue T, Kinoshita H, Komai Y, Kawabata T, Kawa G, Uemura Y and Matsuda T: Two cases of gastrointestinal perforation after radiotherapy in patients receiving tyrosine kinase inhibitor for advanced renal cell carcinoma. World J Surg Oncol 10: 167, 2012.

16. Li XF, Tan YN, Cao Y, Xu JH, Zheng S and Yuan Y: A case report of gastrointestinal hemorrhage and perforation during apatinib treatment of gastric cancer. Medicine (Baltimore) 94: e1661, 2015.

17. Ou SH, Ahn JS, De Petris L, Govindan R, Yang JC, Hughes B Lena H, Moro-Sibilot D, Bearz A, Ramirez SV, et al: Alectinib in crizotinib-refractory ALK-rearranged non-small-cell lung cancer: A phase II global study. J Clin Oncol 34: 661-668, 2016.

18. Gennatas S, Stanway SJ, Thomas R, Min T, Shah R, O'Brien ME and Popat S: Early pneumothorax as a feature of response to crizotinib therapy in a patient with ALK rearranged lung adenocarcinoma. BMC Cancer 13: 207, 2013.

19. Katta A, Fesler MJ, Tan A, Vuong G and Richart JM: Spontaneous bilateral pneumothorax in metastatic renal cell carcinoma on sunitinib therapy. Cancer Chemother Pharmacol 66: 409-412, 2010.

20. Yang SH, Lin JK, Chen WS, Lin TC, Yang SH, Jiang JK, Chang SC, Lan YT, Chao TC, Yen CC, et al: Pneumothorax after bevacizumab-containing chemotherapy: A case report. Jpn J Clin Oncol 41: 269-271, 2011.

21. Zhang Y, Yang H, Zhao M and He X: Bilateral pneumothorax after bevacizumab-containing chemotherapy in fibrosarcoma. J Thorac Dis 4: 229-231, 2012.

22. Makino T, Kudo S and Ogata T: Pneumothorax after treatment with bevacizumab-containing chemotherapy for breast cancer-a case report. Gan To Kagaku Ryoho 41: 233-235, 2014 (In Japanese).

23. Chi Y, Fang Z, Hong X, Yao Y, Sun P, Wang G, Du F, Sun Y, Wu Q, Qu G, et al: Safety and efficacy of anlotinib, a multikinase angiogenesis inhibitor, in patients with refractory metastatic soft-tissue sarcoma. Clin Cancer Res 24: 5233-5238, 2018. 